\title{
Preserving Preclinical PET Quality During Intratherapeutic Imaging in Radionuclide Therapy with Rose Metal Shielding Reducing Photon Flux
}

\author{
Emma Mellhammar ${ }^{1}$, Magnus Dahlbom $^{2}$, Susan Evans-Axelsson ${ }^{2}$, and Sven-Erik Strand ${ }^{1,3}$ \\ ${ }^{1}$ Division of Oncology and Pathology, Department of Clinical Sciences, Lund University, Lund, Sweden; ${ }^{2}$ Department of Molecular \\ and Medical Pharmacology, David Geffen School of Medicine at UCLA, Los Angeles, California; and ${ }^{3}$ Division of Medical Radiation \\ Physics, Department of Clinical Sciences, Lund University, Lund, Sweden
}

\begin{abstract}
Performing PET imaging during ongoing radionuclide therapy can be a promising method to follow tumor response in vivo. However, the high therapeutic activity can interfere with the PET camera performance and degrade both image quality and quantitative capabilities. As a solution, low-energy photon emissions from the therapeutic radionuclide can be highly attenuated, still allowing sufficient detection of annihilation photons in coincidence. Methods: Hollow Rose metal cylinders with walls $2-4 \mathrm{~mm}$ thick were used to shield a ${ }^{22} \mathrm{Na}$ point source and a uniform phantom filled with ${ }^{18} \mathrm{~F}$ as they were imaged on a preclinical PET camera with increasing activities of ${ }^{177} \mathrm{Lu}$. A mouse with a subcutaneous tumor was injected with ${ }^{18} \mathrm{~F}-\mathrm{FDG}$ and imaged with an additional $120 \mathrm{MBq}$ of ${ }^{177} \mathrm{Lu}$ and repeated with shields surrounding the animal. Results: The addition of ${ }^{177} \mathrm{Lu}$ to the volume imaged continuously degraded the image quality with increasing activity. The image quality was improved when shielding was introduced. The shields showed a high ability to produce stable and reproducible results for both spatial resolution and quantification of up to $120 \mathrm{MBq}$ of ${ }^{177} \mathrm{Lu}$ activity (maximum activity tested). Conclusion: Without shielding, the activity quantification will be inaccurate for time points at which therapeutic activities are high. The suggested method shows that the shields reduce the noise induced by the ${ }^{177} \mathrm{Lu}$ and therefore enable longitudinal quantitative intratherapeutic imaging studies.
\end{abstract}

Key Words: PET; radionuclide therapy; count losses; shielding; small-animal imaging

J Nucl Med 2019; 60:710-715

DOI: 10.2967/jnumed.118.217117

$\mathbf{T}$ o improve cancer therapy, there is a need for sensitive and quantitative molecular imaging techniques to follow therapeutic efficacy and progress of disease $(1,2)$.

In preclinical research, molecular imaging techniques such as PET help decipher the response to treatment in animal tumor models. Changes in proliferation, hypoxia, angiogenesis, and other factors important to tumor growth and survival can be monitored in vivo with PET tracers (2-5). PET adds physiologic

\footnotetext{
Received Jul. 3, 2018; revision accepted Oct. 5, 2018.

For correspondence or reprints contact: Emma Mellhammar, Division of Oncology and Pathology, Department of Clinical Sciences, Barngatan 3, SE-221 85 Lund, Sweden.

E-mail: emma.mellhammar@med.lu.se

Published online Nov. 2, 2018.

COPYRIGHT (C 2019 by the Society of Nuclear Medicine and Molecular Imaging.
}

information that can have better prognostic value than measuring tumor size and volume with calipers or with morphologic imaging such as CT or MRI (6). PET tracers enable longitudinal studies, allowing measurements of both early and late treatment response (7).

Radionuclide therapy (RNT) is an expanding field of systemic therapies in which tumor-targeting molecules are labeled with radionuclides to deliver localized radiotherapy to tumor cells with high specificity. ${ }^{177} \mathrm{Lu}$-DOTATATE is a recent effective RNT for the treatment of neuroendocrine tumors, and several other promising treatments are currently under development $(8,9)$, such as ${ }^{177} \mathrm{Lu}$-prostate-specific membrane antigen treatment for metastatic prostate cancer (10-12). Radionuclides used for RNT are chosen for their mode of decay and the linear energy transfer of the emitted particles. To follow response markers in vivo with PET can be both a step toward more personalized medicine and a tool to broaden the radiobiologic understanding of RNT.

However, photon emissions from RNT radionuclides can interfere during intratherapeutic PET. ${ }^{177} \mathrm{Lu}$ has $\gamma$-emissions of 113 and $208 \mathrm{keV}$, with emission yields of $6.2 \%$ and $10.4 \%$, respectively (13). This excess photon flux risks the addition of noise and distortions. Although the low-energy photons can be rejected by energy discrimination, the detector electronics must still process these events. The result may be dead-time losses and loss in sensitivity. Accepted single events from the photon background are also likely to generate a significant amount of random coincidences that will add noise to the image once subtracted. A large photon flux is also likely to result in pulse pileup, which might interfere with the detection of the annihilation photons and will affect the spatial resolution $(14,15)$. We examined this problem on several preclinical PET scanners (16) and found that some systems have limited abilities in handling a high background photon flux from a therapeutic source and display a reduced spatial resolution and image artifacts. In a longitudinal study of an animal undergoing an RNT, where the therapeutic activity will decay from high to low over time, the quantification of the therapeutic effect measured with PET will be uncertain.

We propose a metal shield that surrounds the animal during intratherapeutic PET imaging. The attenuation differs such that lower-energy photons will be more attenuated than the $511-\mathrm{keV}$ photons. Transmission through a Rose metal shield as a function of shield thickness calculated from mass attenuation factors for Rose metal (17) are presented in Figure 1.

Absorbing shields have previously been used in PET imaging. Muehllehner (18) used a graded absorber to attenuate $\gamma$-rays scattered in the patient. This was followed up by Spinks and Shah 


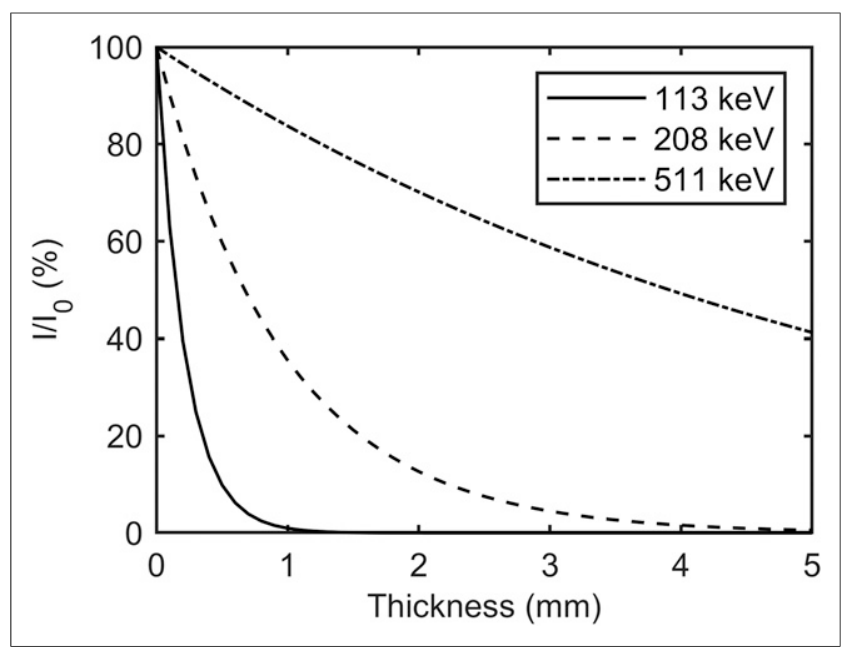

FIGURE 1. The number of transmitted photons I through Rose metal shield in terms of the number without a shield $I_{0}$ for relevant photon energies representing $\mathrm{y}$-emissions from ${ }^{177} \mathrm{Lu}$ and $511-\mathrm{keV}$ annihilation photons.

(19), who evaluated the effect of lead filters on a neuro-PET system. Goertzen et al. investigated the opportunity to perform simultaneous PET and CT by shielding the PET detector with lead (20).

In this paper, image quality is evaluated in a manner similar to that performed by Goertzen et al. evaluating small-animal PET systems (21). They followed the National Electrical Manufacturers Association 4-2008 protocol that standardizes how to report preclinical PET imaging parameters (22). We report similar measurements with a uniform volume of ${ }^{18} \mathrm{~F}$ and a ${ }^{22} \mathrm{Na}$ point source. We also show in vivo proof of concept in a xenografted mouse. Additionally, Monte Carlo simulations are performed to evaluate the count rate effects of shields and energy window discrimination.

\section{MATERIALS AND METHODS}

\section{Shields}

The attenuation of low-energy photons was achieved with cylindric shields of Rose metal. Rose metal is an alloy consisting of $50 \%$ bismuth, $25 \%-28 \%$ lead, and $22 \%-25 \%$ tin. It has a low melting point and does not contract after cooling, making it suitable for custommade radiation shields. It is also less toxic than lead, favoring its use (23). Three cylinders of Rose metal were constructed with an inner diameter of $35 \mathrm{~mm}$ and a wall thickness of 2, 3, or $4 \mathrm{~mm}$ and were all $9 \mathrm{~cm}$ long (Fig. 2).

\section{PET Imaging and Image Evaluation}

The preclinical G8 PET/CT system (Sofie BioSciences, PerkinElmer) (24), the successor of the preclinical PET/CT system Genisys 4 (Sofie BioSciences) (25), was used in this study. The G8 applies a dead-time correction to the data; however, it is accurate only up to a photon flux produced by a mouse injected with $3 \mathrm{MBq}$ of ${ }^{18} \mathrm{~F}-\mathrm{FDG}$.

To run imaging procedures with the shield surrounding the phantom or mouse, a plastic sheet was constructed and used in place of the system's ordinary animal bed. The system's default energy window of $150-650 \mathrm{keV}$ was used in all measurements. All acquisitions were reconstructed as static frames with the system's maximum-likelihood expectation-maximization (60 iterations) algorithm.

VivoQuant (version 3.0; inviCRO) was used to select representative images and to draw volumes of interest (VOIs) over regions in the phantom studies of the reconstructed DICOM images. MATLAB
(R2017b; MathWorks) was used to select line profiles through the ${ }^{22} \mathrm{Na}$ point source in the images and to calculate the full width at half maximum (FWHM) for the axial, coronal, and sagittal projections. The volume FWHM was calculated as the product of the axial, sagittal, and coronal FWHMs.

\section{Count Rate Characteristics}

A $70-\mathrm{kBq}^{22} \mathrm{Na}$ point source (half-life, $2.6 \mathrm{y}$ ) was imaged together with $0.1-50 \mathrm{MBq}$ of $99 \mathrm{~m} \mathrm{Tc}$-sodiumpertechnetate (Triad Isotopes Inc.) dissolved in water in a 1-mL syringe. The point source was placed at the center of the FOV on a stand; the syringe was centered along the transversal axis, just below the point source. The acquisition was made as a dynamic scan, acquiring data for 5 min every hour until the initial $50 \mathrm{MBq}$ of ${ }^{99 m} \mathrm{Tc}$ activity had decayed to less than $0.1 \mathrm{MBq}$. The count rates of coincidence events (prompt, delayed, and true events) for the 5-min acquisitions were retrieved from the histogram file.

The low-energy $\gamma$-photons emitted from ${ }^{99 \mathrm{~m}} \mathrm{Tc}(141 \mathrm{keV})$ adds a background photon flux that will interact in the PET detectors, simulating the presence of a therapeutic radionuclide in the PET camera. The short half-life of $6.0 \mathrm{~h}$ of ${ }^{99 \mathrm{~m}} \mathrm{Tc}$, compared with $6.7 \mathrm{~d}$ for ${ }^{177} \mathrm{Lu}$, allowed a broad photon flux range to be evaluated within a reasonable time frame. Emission branching leads to about a factor of 5 times higher photon flux per decay from ${ }^{99 \mathrm{~m}} \mathrm{Tc}$ than from ${ }^{177} \mathrm{Lu}$.

\section{Point Source}

The ${ }^{22} \mathrm{Na}$ point source was placed on a stand at the center of the field of view (FOV) together with a spheric hollow phantom (Hollow Sphere Sets, $0.5 \mathrm{~mL}$; Data Spectrum) filled with $0-120 \mathrm{MBq}$ of ${ }^{177} \mathrm{Lu}$ ( ${ }^{77} \mathrm{Lu}$-DOTATATE; Advanced Accelerator Applications) dissolved in $0.5 \mathrm{~mL}$ of water. The ${ }^{177} \mathrm{Lu}$ sphere phantom (outer diameter, $1.2 \mathrm{~cm}$ ) was placed at the foot of the stand, approximately $1 \mathrm{~cm}$ below the point source. This setup was imaged in 5-min static acquisitions without and with increasing thickness of shields $(2-4 \mathrm{~mm})$ surrounding both point source and sphere phantom. The volume FWHM of the reconstructed point source was evaluated. The prompt coincidence count rates were extracted from the histogram files to evaluate the effect of the ${ }^{177} \mathrm{Lu}$ background on the coincidence count rate. A spheric VOI with $7.3-\mathrm{mm}$ diameter $\left(200 \mathrm{~mm}^{3}\right)$ was placed over the ${ }^{22} \mathrm{Na}$ point source in the reconstructed images. Image counts in the VOIs were summed and normalized by the summed VOI image counts from the point source when measured without any shield in place or ${ }^{177} \mathrm{Lu}$ activity present in the FOV.

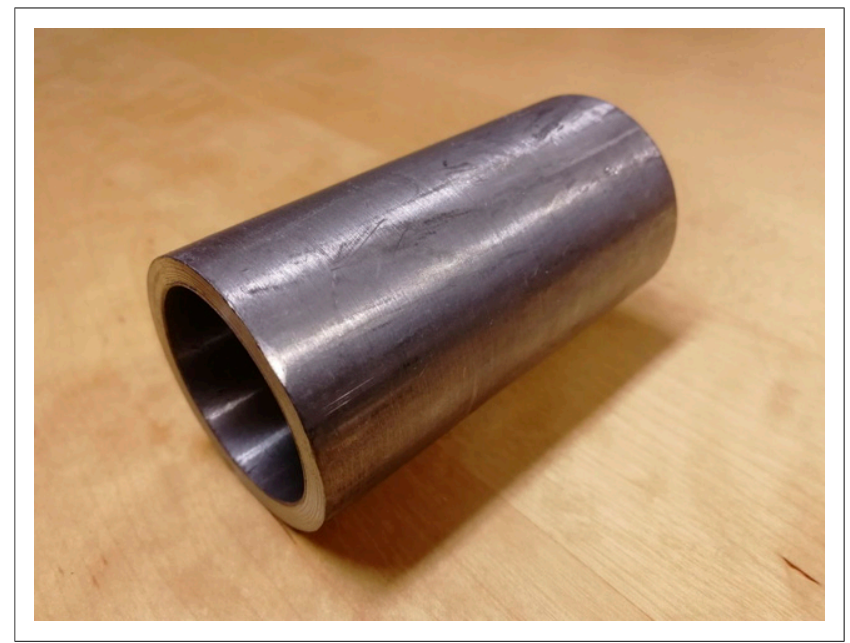

FIGURE 2. Shield of Rose metal for intratherapeutic PET imaging of mice during RNT. Shields used were 2, 3, and $4 \mathrm{~mm}$ thick (here shown with 4-mm thickness). All had inner diameter of $35 \mathrm{~mm}$. 


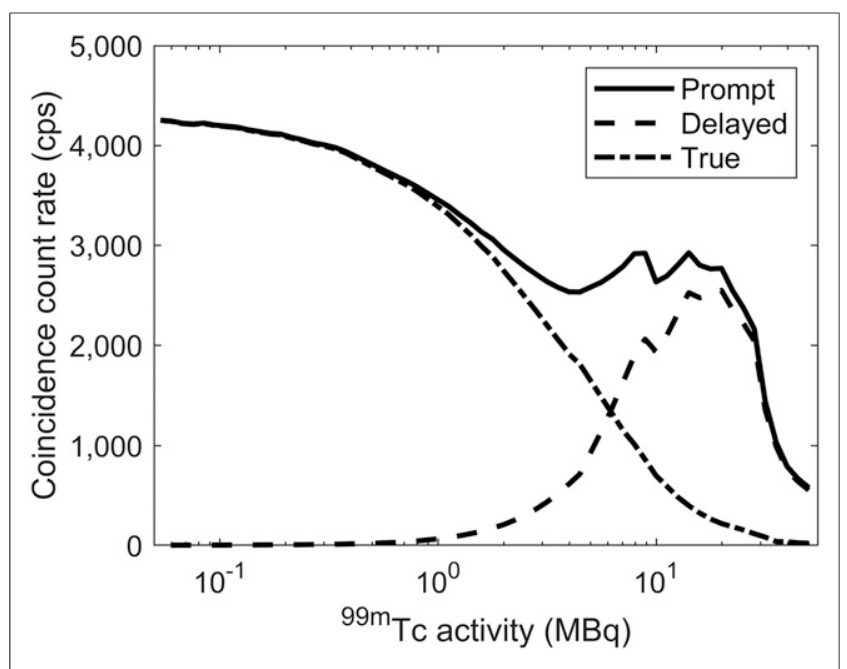

FIGURE 3. Coincidence counts detected in 5-min time frames at beginning of every hour as ${ }^{99 \mathrm{~m} T c}$-filled syringe was imaged together with $70-\mathrm{kBq}^{22} \mathrm{Na}$ point source. As ${ }^{99 \mathrm{mT}} \mathrm{T}$ decayed, broad interval $(>0.1-50$ $\mathrm{MBq}$ ) of low-energy photon flux was examined to evaluate effect on coincidence count rate. Dead-time losses to true coincidence count rate increased as ${ }^{99 \mathrm{~m} T c}$ activity increased.

\section{Uniform ${ }^{18} \mathrm{~F}$ Phantom}

A 20-mL syringe (inner diameter, $21.2 \mathrm{~mm}$ ) filled with $790 \mathrm{kBq}$ of ${ }^{18} \mathrm{~F}$ FDG (produced at the Biomedical Cyclotron Facility at UCLA) was centered along the transversal axis and imaged alone and together with $0-120 \mathrm{MBq}$ of ${ }^{177} \mathrm{Lu}$ in the $0.5-\mathrm{mL}$ sphere phantom placed on top of the syringe at its middle point. Static 10-min acquisitions were made without the shields, and then with the phantoms placed inside shields of increasing thickness $(2-4 \mathrm{~mm})$. A cylindric VOI $\left(710 \mathrm{~mm}^{3}\right)$ was centered inside the ${ }^{18} \mathrm{~F}$ syringe phantom in the reconstructed images. The relative SD (RSD) (coefficient of variation) of the image counts in the VOI was calculated as previously reported $(21,22)$. The sum of image counts detected in the VOI was normalized by the events detected from the ${ }^{18} \mathrm{~F}$ phantom measured without any shield or ${ }^{177} \mathrm{Lu}$ activity present in the FOV.

\section{Mouse ${ }^{18}$ F-FDG Imaging}

The animal experiment was approved by the UCLA Animal Research Committee and was performed according to the guidelines of the Division of Laboratory Animal Medicine at UCLA.
One C57BL/6 mouse bearing a RM1-PGLS-based subcutaneous tumor in the shoulder region was injected with $790 \mathrm{kBq}$ of ${ }^{18} \mathrm{~F}-$ FDG. After $1 \mathrm{~h}$, the mouse was euthanized and repeatedly imaged in 10-min static acquisitions: first by itself with no ${ }^{177} \mathrm{Lu}$ present and then with the spheric phantom containing $120 \mathrm{MBq}$ of ${ }^{177} \mathrm{Lu}$ taped to the right side of its back. Lastly, the animal and the ${ }^{177} \mathrm{Lu}$ source were imaged with each of the shields $(2-4 \mathrm{~mm})$.

\section{Monte Carlo Simulations of the PET System and Shielding}

To get a better understanding of the origin of the image distortions during intratherapeutic PET imaging, spectra of the energy depositions in the PET camera's bismuth germanate detectors were simulated with GATE 8.0, a software for Monte Carlo simulation of medical imaging and radiotherapy (26). Simulating the shields also clarifies the underlying attenuation effects of our method's performance. A model of the Genisys4 preclinical PET system, whose detectors have similar properties and geometry to the G8 PET/CT system, and a cylindric phantom filled with $10 \mathrm{MBq}$ of ${ }^{177} \mathrm{Lu}$ in water (radius, $1 \mathrm{~cm}$; height, $5 \mathrm{~cm}$ ) and a point source of $100 \mathrm{kBq}$ of ${ }^{18} \mathrm{~F}$ placed at the center of the cylindric phantom were simulated. Simulations were repeated with a 4-mm-thick Rose metal shield surrounding the cylinder phantom and point source. The energy depositions of the interactions in the detector volumes were recorded. At least $10^{7}$ initial emissions were generated in both simulations.

A digitizer model describing the detection of singles and coincidences among the energy depositions in the detectors was optimized to reproduce measured single and coincidence rates from $20 \mathrm{kBq}$ to $17 \mathrm{MBq}$ of ${ }^{18} \mathrm{~F}$ dissolved in water in a 20 -mL cylindric phantom (Supplemental Figs. 1A and 1B; supplemental materials are available at http://jnm.snmjournals.org). The dead time was estimated to $1,550 \mathrm{~ns}$ from the singles rate, and the model assumed a paralyzable dead-time behavior. The digitizer model was then used to simulate the coincidence rate when $100 \mathrm{kBq}$ of ${ }^{18} \mathrm{~F}$ and $0-30 \mathrm{MBq}$ of ${ }^{177} \mathrm{Lu}$ homogeneously distributed within a cylindric phantom was imaged with wide $(150-650 \mathrm{keV})$ or narrow $(350-650 \mathrm{keV})$ energy windows, with and without a $3-\mathrm{mm}$ Rose metal shield surrounding the phantom.

\section{RESULTS}

\section{Count Rate Characteristics}

The detected count rates from the ${ }^{22} \mathrm{Na}$ point source as a function of ${ }^{99 \mathrm{~m}} \mathrm{Tc}$ activity are shown in Figure 3 . The true coincidence count rate dropped off almost exponentially as a function of increasing ${ }^{99 \mathrm{~m}} \mathrm{Tc}$ activity because of count losses due to the system's dead time, produced by the increasing flux of low-energy photons. At about $3.4 \mathrm{MBq}$ of ${ }^{99 \mathrm{~m}} \mathrm{Tc}$, the true coincidence count rate was reduced to half that generated by the point source itself. At $6.2 \mathrm{MBq}$, the true coincidence count rate was $32 \%(1,321 / 4,251$ counts/ s) and the numbers of true and delayed coincidences were equal, a point often used as a cutoff for when count rates are too high for accurate PET imaging.

\section{Point Source}

The volume spatial resolution of the ${ }^{22} \mathrm{Na}$ point source as a function of increasing activities of ${ }^{177} \mathrm{Lu}$ in the FOV is presented in Figure 4A and shows an increasing volume FWHM with increasing ${ }^{177} \mathrm{Lu}$ activity. The use of shields recovered the volume 
FWHM to approximately the same spatial resolution as measured for the ${ }^{22} \mathrm{Na}$ point source alone.

The prompt count rates show the attenuating differences between the shields in Figure 4B. The prompt coincidence count rate increased slightly for all shields for increased ${ }^{177} \mathrm{Lu}$ activity, but with a reduced effect for increasing thickness. Without shielding, the prompt coincidences reached a maximum count rate at 40 $\mathrm{MBq}$ of ${ }^{177} \mathrm{Lu}$. As the ${ }^{177} \mathrm{Lu}$ activity was further increased, deadtime losses reduced the coincidence count rate.

The image counts detected in the VOI around the point source are presented in Figure 4C. The signal fell off almost exponentially as the ${ }^{177} \mathrm{Lu}$ activity was increased. The data in Figure $4 \mathrm{C}$ show the reduction in number of coincidences detected because of the attenuation of the shields, which results in an effective sensitivity reduction of the system. The mean and SD of the normalized sum of image counts over the ${ }^{177} \mathrm{Lu}$ interval of 0 $120 \mathrm{MBq}$ for the $4-\mathrm{mm}$ shield was $25 \% \pm 1 \%$. This large reduction in image counts results in an increase in image noise or requires increased imaging time. The reduction will require a new calibration factor for quantitative measurement of the PET tracer. However, the low variation in counts over the interval of $0-120 \mathrm{MBq}$ shows that reliable quantitative intratherapeutic studies are possible to perform.

\section{Uniform ${ }^{18} \mathrm{~F}$ Phantom}

The image quality of a cylindric phantom with a uniform distribution of $790 \mathrm{kBq} \pm 40 \mathrm{kBq}$ (mean $\pm \mathrm{SD}$ ) of ${ }^{18} \mathrm{~F}$ activity was clearly reduced when an increasing amount of ${ }^{177} \mathrm{Lu}$ activity was introduced to the FOV, as demonstrated by the axial images in Figure 5A. The use of shields restored the expected volume distribution of ${ }^{18} \mathrm{~F}$ inside the uniform phantom (Fig. 5A, image collected using a 2-mm shield).

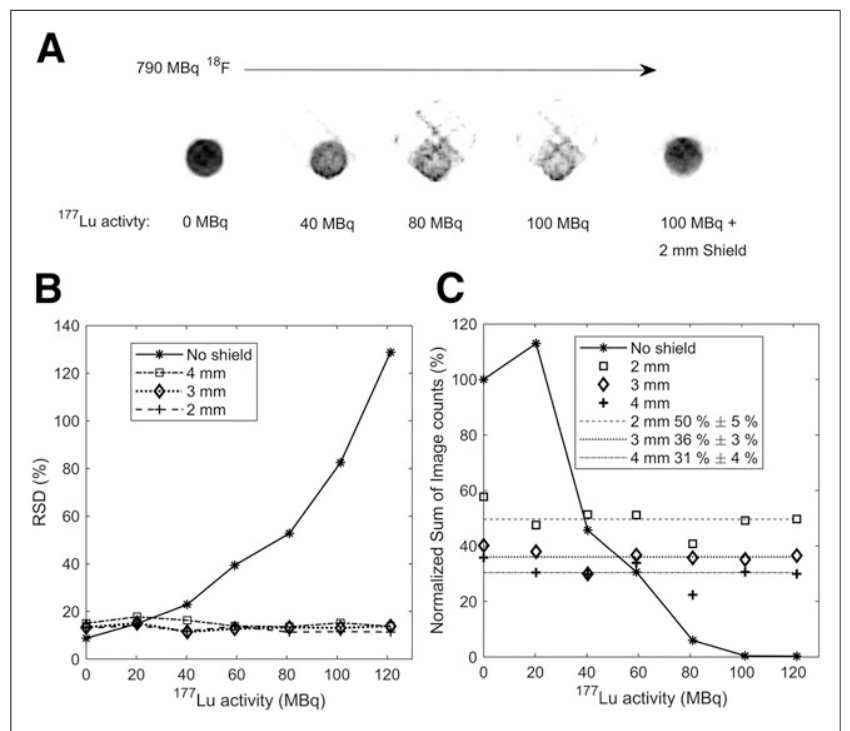

FIGURE 5. Results from $790-\mathrm{kBq}^{18} \mathrm{~F}$ in uniform phantom imaged with 0 - to $120-\mathrm{MBq}^{177} \mathrm{Lu}$ in spheric phantom and 0-, 2-, 3-, and 4-mm Rose metal shields surrounding activity. (A) Axial projections at centrum of uniform phantom filled with $790 \mathrm{kBq}$ of ${ }^{18} \mathrm{~F}$ together with $0,40,80$, and $100 \mathrm{MBq}$ of ${ }^{177} \mathrm{Lu}$. In final image, 2-mm Rose shield was added. (B) RSD of image counts measured with cylindric VOI inside ${ }^{18} \mathrm{~F}$-filled phantom. (C) Normalized sum of image counts detected in cylinder VOI in ${ }^{18} \mathrm{~F}$ phantom. Mean and SD for 0 - to $120-\mathrm{MBq}$ measurements with shields are presented.

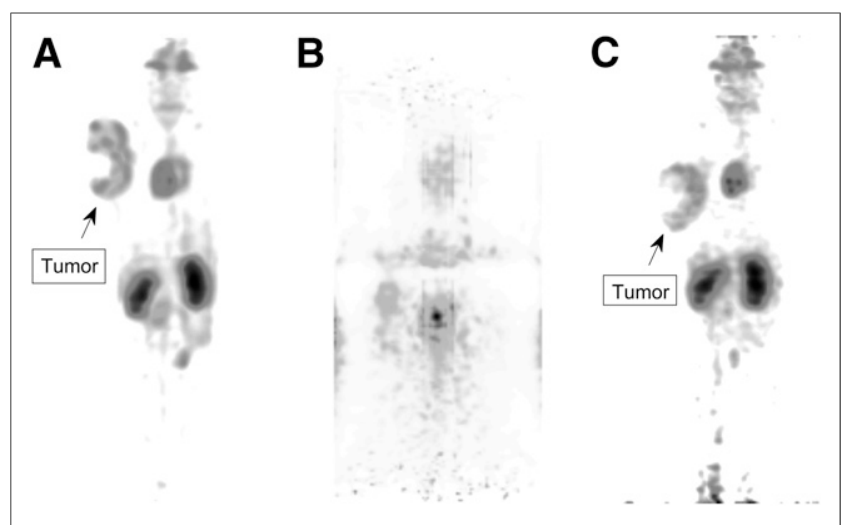

FIGURE 6. Maximum-intensity projections of mouse injected with 790 $\mathrm{kBq}$ of ${ }^{18} \mathrm{~F}-\mathrm{FDG}$ and imaged in regular setup (A), with $120 \mathrm{MBq}$ of ${ }^{177} \mathrm{Lu}$ in spheric phantom taped to flank (B), and with 4-mm Rose metal shield surrounding mouse and ${ }^{177} \mathrm{Lu}$ phantom (C).

The RSD of image counts inside the uniform volume with ${ }^{18} \mathrm{~F}$ as a function of ${ }^{177} \mathrm{Lu}$ activity in the FOV is shown in Figure 5B. Without a shield, the RSD increases with increasing ${ }^{177} \mathrm{Lu}$ activity. In the absence of ${ }^{177} \mathrm{Lu}, \mathrm{RSD}$ increases from $9 \%$ without shielding to $13 \%-15 \%$ with the shields in place. This small increase in noise is expected because of the attenuation of counts in the shields and results in a slight reduction in image quality and increasing variance of a quantitative measurement. However, the presence of the shields results in an RSD that is relatively constant within the range of ${ }^{177} \mathrm{Lu}$ activities investigated $(11 \%-18 \%)$.

The normalized sum of ${ }^{18} \mathrm{~F}$ image counts in the cylindric VOI is presented in Figure 5C. Without shields and with increasing ${ }^{177} \mathrm{Lu}$ activity present, the system lost the ability to reproduce a constant number of image counts in the VOI, but once the shielding was added, repeated measurements over the range of ${ }^{177} \mathrm{Lu}$ activity tested were able to reproduce the image counts, thereby again demonstrating the possibility to perform quantitative studies over a range of ${ }^{177} \mathrm{Lu}$ activities present in the FOV.

\section{Mouse ${ }^{18} \mathrm{~F}-\mathrm{FDG}$ imaging}

Figure 6 shows maximum-intensity projections from a mouse injected with $790 \mathrm{kBq}$ of ${ }^{18} \mathrm{~F}$-FDG and repeatedly imaged $1 \mathrm{~h}$ after injection. Figure $6 \mathrm{~A}$ with no shielding or ${ }^{177} \mathrm{Lu}$ activity added shows only ${ }^{18}$ F-FDG uptake. Expected uptake in heart, kidney, and subcutaneous tumor in the right shoulder area was observed. In Figure $6 \mathrm{~B}, 120 \mathrm{MBq}$ of ${ }^{177} \mathrm{Lu}$ activity in a sphere phantom was taped to the animal's right flank. As can be seen in this image, the ${ }^{18} \mathrm{~F}-\mathrm{FDG}$ distribution is completely distorted in the presence of ${ }^{177} \mathrm{Lu}$. In Figure $6 \mathrm{C}, 4 \mathrm{~mm}$ of Rose metal shielding was placed around the mouse and the ${ }^{177} \mathrm{Lu}$ phantom. The ${ }^{18} \mathrm{~F}-\mathrm{FDG}$ distribution in the mouse when imaged with the Rose metal shields is now restored and comparable to the image in 6A. With the shielding in place, the ${ }^{18} \mathrm{~F}-\mathrm{FDG}$ uptake in the subcutaneous tumor on the shoulder and the heart and kidneys was again clearly distinguishable.

\section{Monte Carlo Simulation of PET System and Shielding}

The energy deposited in the bismuth germanate detectors by a ${ }^{18} \mathrm{~F}$ point source inside a ${ }^{177} \mathrm{Lu}$-filled water phantom was simulated with the GATE 8.0 Monte Carlo simulation toolkit. Without the shield, the rate of energy deposition interactions from the 10$\mathrm{MBq}{ }^{177} \mathrm{Lu}$ phantom and $100-\mathrm{kBq}{ }^{18} \mathrm{~F}$ point source (including 

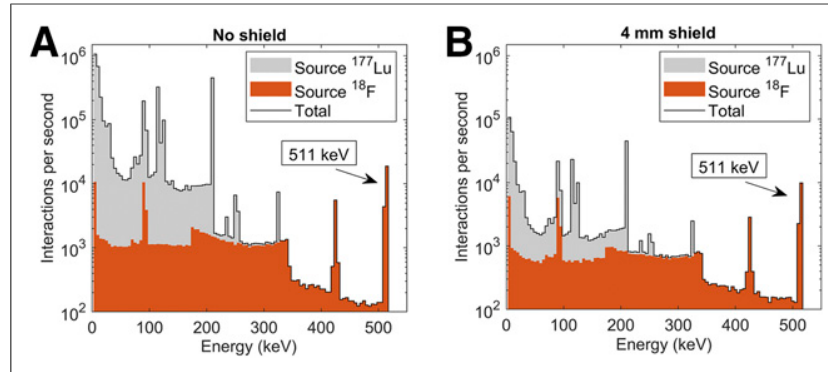

FIGURE 7. Energy deposited in detectors, as simulated by GATE (version 8.0) Monte Carlo simulation toolkit. A $100-\mathrm{kBq}{ }^{18} \mathrm{~F}$ point source centered inside water-filled cylindric phantom with $10 \mathrm{MBq}$ of ${ }^{177} \mathrm{Lu}$ homogeneously distributed inside it were set up inside PET camera FOV. (A) Simulation without shield. (B) Simulation with 4-mm Rose metal shield surrounding phantom and sources.

secondary scattered events) was $3.87 \times 10^{7}$ per second. Of these, $3.73 \times 10^{7}$ per second originated from the ${ }^{177} \mathrm{Lu}$ and $0.14 \times 10^{7}$ per second from ${ }^{18} \mathrm{~F}$. Adding a 4-mm Rose metal shield lowered the rate of interactions to $4.22 \times 10^{6}$ per second, and the interactions originating from ${ }^{177} \mathrm{Lu}$ and ${ }^{18} \mathrm{~F}$ were reduced to $3.42 \times$ $10^{6}(9.2 \%)$ and $0.81 \times 10^{6}(57.9 \%)$, respectively. Figure $7 \mathrm{~A}$ is a histogram of these photon interactions of energy deposition in the PET detectors. The low-energy interactions $(<511 \mathrm{keV})$ dominate, explaining the dead-time losses seen in Figures 3 and $4 \mathrm{C}$, where radionuclides emitting low-energy photons contributed high photon flux interacting in the detectors. In Figure 7B, interactions from the simulation run with a 4-mm Rose metal shield surrounding the phantom are shown. The low-energy interactions from ${ }^{177} \mathrm{Lu}$ are reduced to $9.2 \%$, but the shield still allows $57.9 \%$ of the annihilation photons from the ${ }^{18} \mathrm{~F}$ source to be detected.

The digitizer model showed good agreement with measured single and coincidence count rates (Supplemental Figs. 1A and 1B). Simulating increasing ${ }^{177} \mathrm{Lu}$ activity present in the phantom volume continuously lowered the prompt coincidence rates for both the wide (150-650 keV) and the narrow (350-650 keV) energy windows (Supplemental Fig. 1C). The random coincidence rate was significantly reduced using the narrow window. The decrease in prompt rate with increased ${ }^{177} \mathrm{Lu}$ activity is still observed, as this is due to dead-time losses at high count rates. Simulating a 3-mm Rose shield around the phantom showed that the prompt coincidence rate was less affected by increasing ${ }^{177} \mathrm{Lu}$ activity for both energy windows.

\section{DISCUSSION}

In recent years, new models of small preclinical PET cameras have been introduced on the market. These systems can be influential in the development of new RNTs, offering an in vivo opportunity to follow treatment effect in tumor models. The sensitivity to overall system dead-time losses will be a problem in small preclinical systems, which often excel in high sensitivity (21). Fortunately, with shielding, the system's high sensitivity is also a helpful factor when the signal from the PET tracer, inevitably reduced by the shield, still can be successfully detected as shown in this study. Also, injected PET tracer activity could potentially be increased to compensate for the signal loss. The attenuation of annihilation photons in the shields will also result in an increase in scattered photons, which may affect quantification. A narrow energy window could compensate.
The addition of ${ }^{177} \mathrm{Lu}$ activities relevant in preclinical RNT (27) reduced the spatial resolution as seen in Figure 4A, increased the noise, and obscured the images beyond the point at which they could contribute useful and quantifiable information, as shown in Figures $5 \mathrm{C}$ and $6 \mathrm{~B}$. The shields produced a consistent image resolution that was unaffected by the ${ }^{177} \mathrm{Lu}$ activity interval tested. The detected activity in uniform volumes of ${ }^{18} \mathrm{~F}$ remained constant within the investigated activity range of ${ }^{177} \mathrm{Lu}$ activities in the FOV (Figs. 4C and 5B). The use of the shields would therefore allow quantitative imaging and longitudinal studies. The reduction in sensitivity could be compensated by increasing the acquisition time.

The simulated effects of the shields are presented in Figure 7. Rose metal attenuates the photons from ${ }^{177} \mathrm{Lu}$ to a higher degree than the 511-keV annihilation photons. Reducing detected events from ${ }^{177} \mathrm{Lu} \gamma$-emissions lowers the total detector interaction count rate and reduces dead-time losses of true annihilation photon events. Simulations also showed how count losses due to high count rates could not be compensated for using a narrow energy window (Supplemental Fig. 1C). However, for systems tolerating higher count rates or with lower sensitivity, the use of a narrow energy window can be a component in optimization for intratherapeutic PET.

Despite the slight loss of image quality, PET imaging during RNT with shielding can provide clear images of a PET tracer in vivo, as shown in Figure $6 \mathrm{C}$, and makes quantitative measures possible. Our proposed solution makes RNT intratherapeutic PET imaging a promising, easy, and inexpensive method to overcome the deteriorating effect.

\section{CONCLUSION}

Performing preclinical PET imaging during RNT can lead to loss of image quality and distorted quantitative measurements. Shielding makes intratherapeutic PET imaging possible, even with highly sensitive preclinical block detector PET systems. If the effects of a therapeutic radionuclide present in the animal are not accounted for, quantitative studies can underestimate the uptake of a PET tracer because of count losses at high detector dead time. For longitudinal studies of animal models undergoing RNT, this will distort data of activity uptake as the therapeutic radionuclide decays over time.

\section{DISCLOSURE}

Financial support was provided by the Swedish Cancer Society and Mrs. Berta Kamprad's Foundation. Susan Evans-Axelsson was supported by a Swedish Research Council International postdoc grant (grant 2015-00452). No other potential conflict of interest relevant to this article was reported.

\section{ACKNOWLEDGMENTS}

Thanks to Zheng Gu (Crump Institute, UCLA) for providing the GATE model. Thanks to Lars Ljus and the staff at the Radiation Therapy Unit workshop at Skane University Hospital Lund for help constructing the shields.

\section{REFERENCES}

1. Timpson P, McGhee EJ, Anderson KI. Imaging molecular dynamics in vivo: from cell biology to animal models. J Cell Sci. 2011;124:2877-2890.

2. Fernandes RS, de Aguiar Ferreira C, Soares DCF, et al. The role of radionuclide probes for monitoring anti-tumor drugs efficacy: a brief review. Biomed Pharmacother. 2017;95:469-476. 
3. Kelada OJ, Carlson DJ. Molecular imaging of tumor hypoxia with positron emission tomography. Radiat Res. 2014;181:335-349.

4. Rajendran JG, Krohn KA. F-18 fluoromisonidazole for imaging tumor hypoxia: imaging the microenvironment for personalized cancer therapy. Semin Nucl Med. 2015;45:151-162.

5. Tomasi G, Rosso L. PET imaging: implications for the future of therapy monitoring with PET/CT in oncology. Curr Opin Pharmacol. 2012;12:569-575.

6. Kim JH, Kim BJ, Jang HJ, Kim HS. Comparison of the RECIST and EORTC PET criteria in the tumor response assessment: a pooled analysis and review. Cancer Chemother Pharmacol. 2017;80:729-735.

7. de Jong M, Essers J, van Weerden WM. Imaging preclinical tumour models: improving translational power. Nat Rev Cancer. 2014;14:481-493.

8. Norain A, Dadachova E. Targeted radionuclide therapy of melanoma. Semin Nucl Med. 2016;46:250-259.

9. Gill MR, Falzone N, Du Y, Vallis KA. Targeted radionuclide therapy in combined-modality regimens. Lancet Oncol. 2017;18:e414-e423.

10. Eiber M, Fendler WP, Rowe SP, et al. Prostate-specific membrane antigen ligands for imaging and therapy. J Nucl Med. 2017;58(suppl):67S-76S.

11. Rahbar K, Ahmadzadehfar H, Kratochwil C, et al. German multicenter study investigating ${ }^{177} \mathrm{Lu}-\mathrm{PSMA}-617$ radioligand therapy in advanced prostate cancer patients. J Nucl Med. 2017;58:85-90.

12. Baum RP, Kulkarni HR, Schuchardt C, et al. ${ }^{177} \mathrm{Lu}$-labeled prostate-specific membrane antigen radioligand therapy of metastatic castration-resistant prostate cancer: safety and efficacy. J Nucl Med. 2016;57:1006-1013.

13. Data Produced Using the MIRD Program, and Extracted from the Evaluated Nuclear Structure Data File (ENSDF). Upton, NY: Brookhaven National Laboratory; May 2003.

14. Germano G, Hoffman E. A study of data loss and mispositioning due to pileup in 2-D detectors in PET. IEEE Trans Nucl Sci. 1990;37:671-675.

15. Badawi RD, Surti S, Kemp B, et al. The effects of pulse pile-up on point-source measurements performed on different PET scanning devices. IEEE Explore website. https://ieeexplore.ieee.org/document/1352418. Published November 8, 2004.
16. Mellhammar E, Dahlbom M, Axelsson J, Strand SE. Counting rate characteristics and image distortion in preclinical PET imaging during radiopharmaceutical therapy. J Nucl Med. 2016;57:1964-1970.

17. Berger MJ, Hubbell JH, Seltzer SM, et al. XCOM: Photon Cross Section Database (Version 1.5). Gaithersburg, MD: National Institute of Standards and Technology; November 2010.

18. Muehllehner G. Positron camera with extended counting rate capability. J Nucl Med. 1975;16:653-657.

19. Spinks TJ, Shah SI. Effect of lead filters on the performance of a neuro-PET tomograph operated without septa. IEEE Trans Nucl Sci. 1993;40:1087-1091.

20. Goertzen AL, Meadors AK, Silverman RW, Cherry SR. Simultaneous molecular and anatomical imaging of the mouse in vivo. Phys Med Biol. 2002;47:43154328.

21. Goertzen AL, Bao Q, Bergeron M, et al. NEMA NU 4-2008 comparison of preclinical PET imaging systems. J Nucl Med. 2012;53:1300-1309.

22. National Electrical Manufacturers Association (NEMA). Performance Measurements of Small Animal Positron Emission Tomographs: NEMA Standards Publication NU 4-2008. Rosslyn, VA: NEMA; 2008:23.

23. Peterson M, Strand SE, Ljungberg M. Using Rose's metal alloy as a pinhole collimator material in preclinical small-animal imaging: a Monte Carlo evaluation. Med Phys. 2015;42:1698-1709.

24. Gu Z, Taschereau R, Vu N, et al. Performance evaluation of G8, a high sensitivity benchtop preclinical PET/CT tomograph. J Nucl Med. June 14, 2018 [Epub ahead of print].

25. Herrmann K, Dahlbom M, Nathanson D, et al. Evaluation of the Genisys4, a bench-top preclinical PET scanner. J Nucl Med. 2013;54:1162-1167.

26. Jan S, Santin G, Strul D, et al. GATE: a simulation toolkit for PET and SPECT. Phys Med Biol. 2004;49:4543-4561.

27. Schüler E, Osterlund A, Forssell-Aronsson E. The amount of injected ${ }^{177} \mathrm{Lu}-$ octreotate strongly influences biodistribution and dosimetry in C57BL/6N mice. Acta Oncol. 2016;55:68-76. 